\title{
First-Principles Calculations of Second-Order Elastic Constants and Generalized-Stacking-Fault Energy for GaAs
}

\author{
Lumei Tong ${ }^{1}$, Yunchang Fu' ${ }^{1}$, , Huili Zhang ${ }^{1, b}$, Chun Zhang ${ }^{1}$ \\ and Chunhua Zeng ${ }^{1}$ \\ ${ }^{1}$ Kunming University of Science and Technology, Kunming 650500, P.R. China \\ afychang_box@hotmail.com, bzhl010195@163.com
}

Keywords: First-principles, SOECs, GSFE, core structure, Peierls stress.

\begin{abstract}
The second-order elastic constants and generalized-stacking-fault energy surfaces for semiconductors GaAs have been predicted by using the first-principles calculations. The calculations employ the density functional theory within local-density-approximation method. The lattice constants of GaAs is $5.626 \AA$, which agree well with the experimental data $5.65 \AA$ and theoretical values $5.6045 \AA(\mathrm{LDA})$. The SOECs agree well with the experimental data and theoretical values. The generalized-stacking-fault energy curves along $\langle 110\rangle\{111\}$ direction of the shuffle set in GaAs have been calculated. Based on the Fourier series, the fitted generalized-stacking-fault energy surfaces have been obtained. The dislocation width and Peierls stress for shuffle $60^{\circ}$ dislocation in GaAs have been calculated by the improved Peierls-Nabarro theory in which non-relaxed and relaxed calculations have been taken into account. The calculated dislocation width is narrow(about $0.83 \mathrm{~b}$ ), and the peierls stress is about $0.45 \sim 0.54 \mathrm{GPa}$.
\end{abstract}

\section{Introduction}

In the middle of last century, the invention of single crystal silicon and semiconductor transistor and the development of silicon integrated circuit, which led to the electronic industrial revolution; At the beginning of the seventies of the last century, the invention of quartz optical fiber materials and GaAs lasers, which promoted the optical fiber communication technology rapid development and gradually formed a high and new technology industry and mankind has entered the information age. The mechanical properties of III-V compounds with a zinc-blende structure such as GaAs has attracted increasing interest. GaAs has the excellent properties that do work at high temperatures compared with $\mathrm{Si}$ and $\mathrm{Ge}$. One of the mechanical properties are elasticity, in the linear theory of elasticity, the second-order elastic constants(SOECs) are sufficient to describe the elastic stress-strain response and wave propagation in solids and many experimental and theoretical research focus on the SOECs of GaAs [1-5]. These results are very vital for understanding the elasticity of GaAs.

The plasticity is another mechanical property of GaAs. It is well known that dislocations have a large effect on the plasticity of material in covalent crystal and the critical shear stress is related to the mobility of the dislocations. The GSFE introduced by Vitek [6, 7] plays a crucial rule in describing accurately the core structures and motion of dislocations accurately [8,9]. And it also gives information about the shear properties of the perfect crystal [10]. In addition, the GSFE surface is important in proposed models for the brittle-ductile transition $[11,12]$. The GSFE surface is the interplaner potential energy when one-half of a crystal sliding over the other half. Although GSFE curves are experimentally not accessible, such a potential can be determined by the interatomic potential simulation methods such as embedded atom method(EAM) and modified EAM [13, 14] and first-principles calculations $[9,15,16]$. The GSFE has been calculated more accurately form first principles by Hartford et al. [9] in comparison with the methods of interatomic potentials. However, the GSFE and mechanical properties of dislocations in GaAs have been less studied.

In this paper, we have calculated the SOECs and GSFE along $\langle 110\rangle\{111\}$ direction of GaAs using the first-principles total-energy calculations. The core structure and Peierls stress of shuffle $60^{\circ}$ dislocation in GaAs have been investigated by the improved Peierls-Nabarro(P-N) theory [17]. In 
next section, the SOECs and GSFE curves in GaAs have been calculated by the first-calculations method. Section 3 focused on the calculation of core structure and Peierls stress by using the improved P-N theory. The last section is the conclusion.

\section{First-Principles Calculations of Elastic Constants and GSFE}

We carry out first-principles total-energy calculations based on the planewave basis projector augmented wave (PAW) method [18, 19] and the density functional theory (DFT), using the Vienna ab initio simulation package(VASP 4.6) program developed at the Institut für Materialphysik of Universität Wien [20, 22]. The exchange-correlation functions for local-density-approximation (LDA) is used. The convergence of energy and force are set to $1.0 \times 10^{6} \mathrm{eV}$ and $1.0 \times 10^{4} \mathrm{eV} / \AA$, respectively. In order to get accurate results, the k-point mesh size with $21 \times 21 \times 21$ and the plane-wave cut-off was set of $500 \mathrm{eV}$ to calculate the SOECs of GaAs, while the GSFE calculations adopt the grid meshes of $21 \times 21 \times 1$. The equilibrium theoretical crystal structures are determined by minimizing the Hellmann-Feynman force on the atoms and the stress on the unit cell. The calculated lattice constants and SOECs of GaAs have been listed in Table 1 along with the available experimental data and other theoretical calculations. Obviously, our calculated lattice constants is $5.626 \AA$, which is agreement with the experimental data 5.65 $\AA$ and theoretical values 5.6045 $($ LDA). There are three independent SOECs because of the cubic symmetry for GaAs, and the previous results and the experimental data are listed in Table 1. The obtained SOECs are agreement with the previous results and the experiment data.

Table 1. Comparison of the calculated lattice constants and SOECs for GaAs with the experimental data and previous calculations. All data are in unit of GPa except of the lattice constant in $\AA$.

\begin{tabular}{|c|c|c|c|}
\hline & Present calculation & Previous calculations & experiments \\
\hline$a_{0}$ & 5.626 & $5.671(\mathrm{GGA})^{\mathrm{a}}, 5.6045(\mathrm{LDA})^{\mathrm{a}}, 5.601^{\mathrm{b}}$ & $5.653^{\mathrm{f}}, 5.65^{\mathrm{g}}$ \\
\hline$C_{11}$ & 117 & $118.5^{\mathrm{a}}, 133.2^{\mathrm{c}}, 126^{\mathrm{d}}, 100^{\mathrm{e}}$ & $118.1^{\mathrm{h}},(118.4 \sim 0.4)^{\mathrm{j}}$ \\
\hline$C_{12}$ & 60 & $54.1^{\mathrm{a}}, 57.4^{\mathrm{c}}, 55^{\mathrm{d}}, 49^{\mathrm{e}}$ & $53.2^{\mathrm{h}},(53.7 \sim 1.5)^{\mathrm{j}}$ \\
\hline$C_{44}$ & 57 & $60.7^{\mathrm{a}}, 61.6^{\mathrm{c}}, 61^{\mathrm{d}}, 52^{\mathrm{e}}$ & $59.2^{\mathrm{h}},(59.1 \sim 0.2)^{\mathrm{j}}$ \\
\hline
\end{tabular}

${ }^{\mathrm{a}} \operatorname{Ref},[2],{ }^{\mathrm{b}} \operatorname{Ref}[23],{ }^{\mathrm{C}} \operatorname{Ref}[3],{ }^{\mathrm{d}} \operatorname{Ref}[4],{ }^{\mathrm{e}} \operatorname{Ref}[5],{ }^{\mathrm{f}} \operatorname{Ref}[24],{ }^{\mathrm{g}} \operatorname{Ref}[1],{ }^{\mathrm{h}} \operatorname{Ref}[25],{ }^{\mathrm{j}} \operatorname{Ref}[26]$

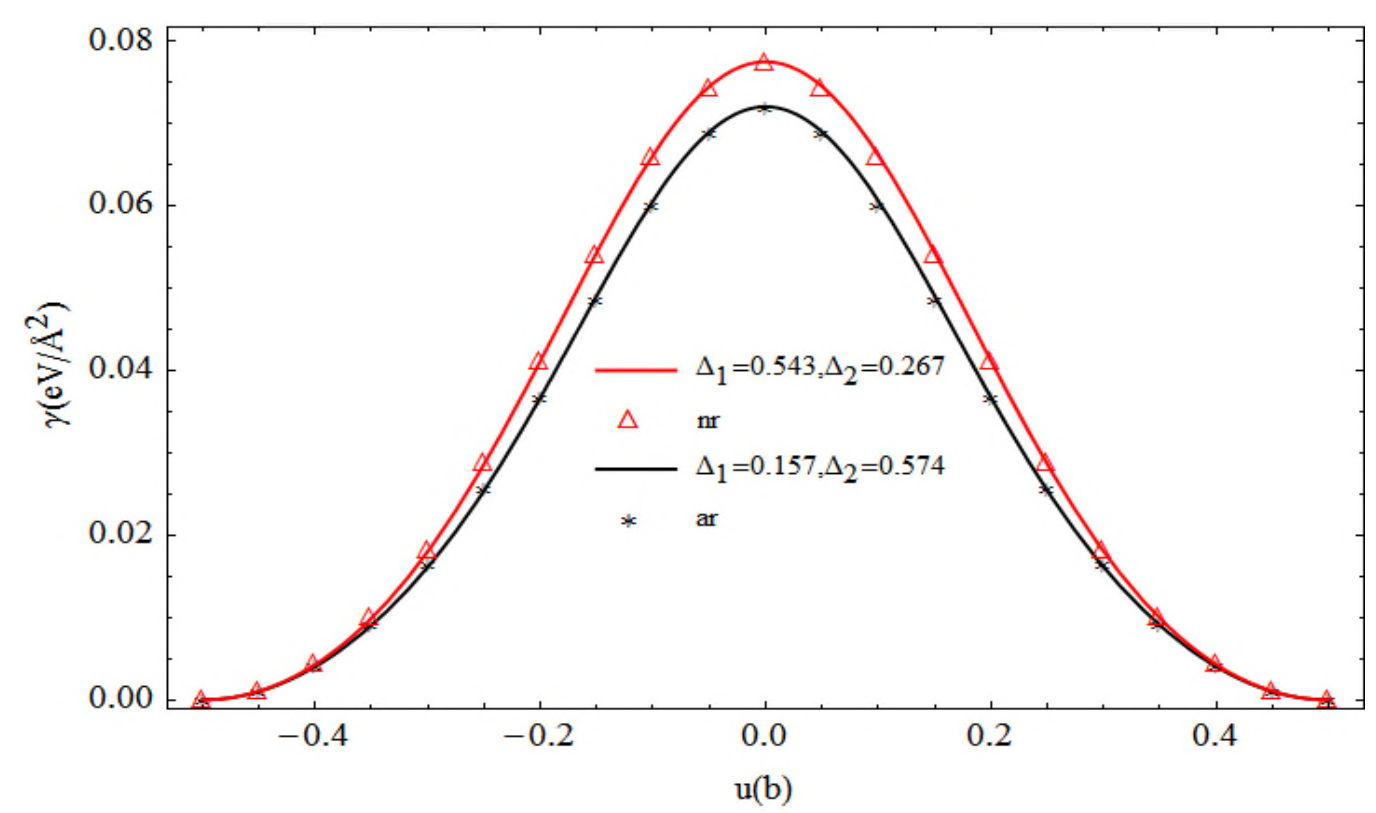

Fig. 1 GSFE curves for GaAs sliding along the $\langle 110\rangle$ direction on the $\{111\}$ plane. The lines denote the fitted curves from Fourier series (Eq. 8), and the discrete points denote the results given by first-principles calculations. 
We calculated the GSFEs of GaAs for $\{111\}$ plane using a slab calculation. A slab consisting of 12 atomic layers in the $\langle\overline{1} 00>$ direction to simulate the stacking-fault process. To avoid the interactions between two slabs, The vacuum space of $15 \AA$ normal to $\{111\}$ plane between periodically repeated slab is chosen. We performed the repeating calculations to obtain the reasonable results, and find that the fluctuations of calculated results for vacuum gap of $15 \AA$ and $18 \AA$ are less than $0.1 \%$. The lattice misfits for the GSFE curves calculation are determined as a difference of total energies for the upper six atomic layers shifting below the slab along $\langle 110\rangle$ direction with non-relaxed and relaxed calculation. For the relaxed calculation, the atoms within the two surfaces of the slab are fixed during the relaxation and for each rigid sliding configuration, the atomic positions are relaxed along the $<\overline{1} 00>$ direction by minimization of the Hellmann-Feynman forces on each atom. The calculated GSFEs along <110>\{110\} direction of the shuffle set for GaAs have been shown in Fig. 1.

\section{Dislocation Equation within Improved P-N Theory}

The two-dimensional dislocation equation can be expressed as [17]

$$
-\frac{\beta}{2} \frac{d^{2} u}{d x^{2}}-\left.\frac{K \sigma}{2 \pi} \int_{-\infty}^{+\infty}\left(\frac{d x^{\prime}}{x^{\prime}-x}\right)\right|_{x-x^{\prime}}=f
$$

where, $u$ is the dislocation field and $\sigma$ is the area of primitive cell of the misfit plane. $K$ is the energy factors of the mixed dislocation. The discrete parameter and energy factor $K$ can been determined as $[17,27]$

$$
\beta=\frac{\left(3 C_{11}+5 C_{12}\right) a_{0}^{3}}{24} \sin ^{2} \theta+\frac{\left(C_{11}-C_{12}\right) a_{0}^{3}}{16} \cos ^{2} \theta, K=\mu\left(\frac{\sin ^{2} \theta}{1-v}+\cos ^{2} \theta\right)
$$

where, $\theta$ is the angle between Burgers and vector dislocation line. The equilibrium lattice constant $a_{0}$ and elastic constants $C_{11}$ and $C_{22}$ for GaAs see Table $1 . \mu$ and $v$ is the effective shear modulus and Poisson's radio in $\{111\}$ surface, they can be obtained

$$
\mu=\sqrt{d_{44} \times C_{44}}, v=1-\frac{\mu}{\varepsilon}
$$

where, $d_{44}$ and $\varepsilon$ can be expressed as

$$
\begin{aligned}
& d_{44}=C_{44}-\frac{h}{2} \\
& \varepsilon=\left(2+\frac{d_{11}}{d_{33}}\right) \times \frac{\left(d_{33}+C_{12}\right)}{3} \sqrt{\frac{C_{44}\left(d_{33}-C_{12}\right)}{d_{11}\left(d_{33}+C_{12}+2 C_{44}\right)}}
\end{aligned}
$$

with

$$
d_{11}=C_{11}+\frac{h}{2}, d_{33}=\sqrt{C_{11} \times d_{11}}
$$

$h$ can be given by

$$
h=2 C_{44}+C_{12}-C_{11}
$$

where $C_{11}, C_{12}$ and $C_{44}$ for GaAs see Table 1 . We can get the $\mu=40 \mathrm{GPa}$ and $\nu=0.32$.

$f$ is the restoring force of atoms along the Burgers vector of mixed dislocation that given by the gradient of the $\gamma$-surface as suggested by Christian and Vitek [28].

$$
f=-\nabla \gamma(u) \sigma
$$


It is noted that the $\gamma$-surfaces along $<110\rangle$ direction of the shuffle set can been approximately expressed as

$$
\gamma(u)=\gamma\left(1+\cos \frac{2 \pi u}{b}\right)\left(1+\Delta_{1} \cos ^{2} \frac{\pi u}{b}+\Delta_{2} \cos { }^{4} \frac{\pi u}{b}\right)
$$

where, $b$ is the Burgers vector, $\gamma, \Delta_{1}$ and $\Delta_{2}$ is given by the unstable stacking fault energy $\gamma_{u s}$ of the modification to the cosine-force law, the fitting curves see Fig. 1.

To solve the dislocation Eq. (1), a new method called the truncating method has been proposed by Wang, which has been verified to be a convenient and efficient method [17]. Only one constant that needs to be determined by this trial solution.

$$
u=\frac{b}{\pi}\left(\arctan q+\frac{c q}{1+q^{2}}\right)
$$

with

$$
q=k x, k=k_{0}(1-c), k_{0}=\frac{d}{2}\left(\frac{\sin ^{2} \theta}{1-v}+\cos ^{2} \theta\right)^{-1}
$$

where, $d$ is the spacing between glide planes and $c$ is a constant determined by the dislocation equation. Following the truncating method with substituting the solution Eq. (9) into Eq. (1), it is found that parameter $c$ should satisfy the algebraic equation.

$$
\frac{3 \beta b^{2} k^{2}}{8 \pi^{2} \gamma \sigma}(5+11 c)+\frac{5 K b^{2} k}{8 \pi^{2} \gamma}(2+3 c)-5(2+c)-3(5+c) \Delta_{1}-18 \Delta_{2}=0
$$

The core parameter $c$ and half width $\xi$ (defined as the distance over which $u$ changes from 0 to $b / 4$ ) of shuffle $60^{\circ}$ dislocation in GaAs are listed in Table 2.

The Peierls stress can be obtained from maximum slope of the dislocation energy

$$
\sigma_{p}=\max \left|\frac{1}{b} \frac{E_{d i s}(x)}{d x}\right|
$$

where, $E_{d i s}(x)$ is the dislocation energy. For a dislocation with length $L$, the dislocation energy per unit length can be expressed as [17]

$$
E_{\text {dis }}(x)=E_{s}(x)+E_{m}(x)
$$

The strain energy and misfit energies of dislocation per unit length can be expressed as

$$
\begin{aligned}
& E_{s}(x)=\sum_{l=-\infty}^{\infty} \frac{u_{l} \sigma}{8 a b} \pi \gamma\left\{8\left(1+\Delta_{1}\right) \sin \frac{2 \pi u_{l}}{b}+3\left(3+\cos \frac{4 \pi u_{l}}{b}\right) \Delta_{2} \sin \frac{2 \pi u_{l}}{b}+2\left(2 \Delta_{1}+3 \Delta_{2}\right) \sin \frac{4 \pi u_{l}}{b}\right\} \\
& E_{m}(x)=\sum_{l=\infty}^{\infty} \frac{\sigma \gamma}{a}\left(1+\cos \frac{2 \pi u_{l}}{b}\right)\left(1+\Delta_{1} \cos 2 \frac{\pi u_{l}}{b}+\Delta_{2} \cos { }^{4} \frac{\pi u_{l}}{b}\right)
\end{aligned}
$$

where, $u_{l}=u\left(x_{l}-x_{0}\right)$ is the relative displacement for dislocation located at $x_{0}$, a is the length of the primitive vector and the sum is carried over the atoms in the horizontal band with width a in a misfit plane (Fig. 2).

The calculated Peierls stresses of shuffle $60^{\circ}$ dislocation in GaAs are also listed in Table 2. 


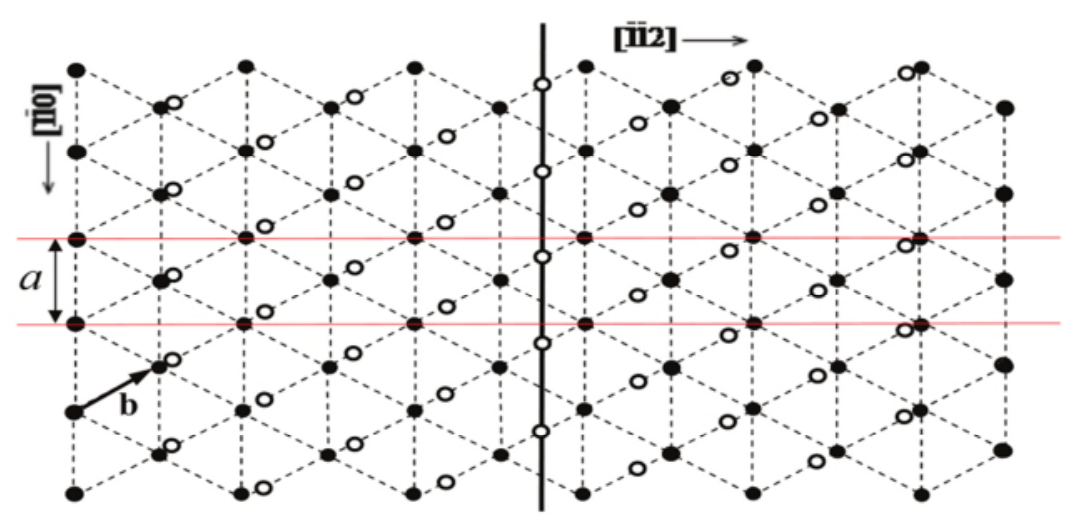

shuffle $60^{\circ}$ dislocation

Fig. 2 Core structure of shuffle $60^{\circ}$ dislocations. The solid and empty circles respectively represent the atoms on the misfit planes that below and above the cut plane.

Table 2. The core parameter $\mathrm{c}$ and half width of shuffle $60^{\circ}$ dislocation in GaAs.

The Peierls stress is in unit of $10^{2} \mathrm{eV} / \AA^{3}$.

\begin{tabular}{|c|c|c|c|c|c|c|}
\hline & $\gamma$ & $\Delta_{1}$ & $\Delta_{2}$ & $\mathrm{c}$ & $\xi$ & $\sigma_{p}$ \\
\hline $\mathrm{ar}$ & 0.0208 & 0.157 & 0.574 & 0.74 & 0.83 & 0.34 \\
\hline $\mathrm{nr}$ & 0.0214 & 0.543 & 0.267 & 0.74 & 0.83 & 0.28 \\
\hline
\end{tabular}

\section{Summary and Discussion}

In this work, the SOECs and GSFE for GaAs are calculated by first-principles total-energy calculations of DFT method. The predictions for SOECs are obtained from the coefficients of the fitted polynomials of the energy-strain functions. The SOECs are agreement well with the experimental data and theoretical values. The GSFE has been obtained with non-relaxed and relaxed calculations, respectively. Combining the calculated SOECs, GSFE and the improved P-N theory, the dislocation width and Peierls stress of shuffle $60^{\circ}$ dislocation in GaAs have been calculated. The calculated dislocation width is narrow(about $0.83 \mathrm{~b}$ ), and it is reasonable for dislocations in covalent crystalline materials. The peierls stress for GaAs is about 0.45 0.54GPa.

\section{Acknowledgement}

This work was supported by the National Natural Science Foundation of China (Grant No.11305079), the introduction of talent capital group fund project of Kunming University of Science and Technology(under KKZ3201407030), the Candidate Talents Training Fund of Yunnan Province (Project Nos. 2015HB025).

\section{References}

[1] J. S. Blakemore, Semiconducting and other major properties of gallium arsenide, J. Appl. Phys. 53, (1982), R123.

[2] S. Saib, N. Bouarissa, High-pressure band parameters for GaAs: first principles calculations, Solid-state electronics. 50(5) (2006) 763-768.

[3] N. Bouarissa and R. Bachiri. Elastic constants and related properties of $\mathrm{AlxGa}_{1-\mathrm{x}} \mathrm{As}_{\mathrm{y}} \mathrm{Sb}_{1-\mathrm{y}} / \mathrm{InAs}$, Phys. B. 322(1-2) (2002) 193-200.

[4] J. Sörgel and U. Scherz, Ab initio calculation of elastic constants and electronic properties of ZnSe and ZnTe under uniaxial strain, Eur. Phys. J. B. 5 (1998)45- 52.

[5] M. Łopuszynski and J. A. Majewski, Ab initio calculations of third-order elastic constants and related properties for selected semiconductors, Phys. Rev. B. 76(4) (2007) 045202. 
[6] V. Vitek, Intrinsic stacking faults in body-centred cubic crystals, Philos. Mag. 18(154) (1968) 773-786.

[7] V. Vitek, Theory of Core Structures of Dislocations in Body-Centered Cubic Metals, Cryst. Lattice Defects. 5(1) (1974)1-34.

[8] B. Jós, Q. Ren and M.S. Duesbery, Peierls-Nabarro model of dislocations in silicon with generalized stacking-fault restoring forces, Phys. Rev. B. 50 (1994) 11882.

[9] J. Hartford, B. von Sydow and G. Wahnstr, B.I. Lundqvist, Peierls barriers and stresses for edge dislocations in Pd and Al calculated from first principles, Phys. Rev. B. 58 (1998) 2487.

[10] C. Brandl, P.M. Derlet, H.V. Swygenhoven, General-stacking-fault energies in highly strained metallic environments: Ab initio calculations, Phys. Rev. B. 76 (2007) 054124.

[11]R. Thomson, Intrinsic ductility criterion for interfaces in solids, Phys. Rev. B. 52 (1995) 7214.

[12] Y. Sun, E. Kaxiras, Slip energy barriers in aluminium and implications for ductile-brittle behaviour, Philos. Mag. A. 75(4) (1997) 1117-1127.

[13] J. M. Zhang, X. J. Wu, Y. J. Huang, K. W. Xu, Energy calculation of the stacking fault in fcc metals by embedded-atom method, Acta Phys. Sin. 55(1) (2006) 393-397.

[14] X. M. Wei, J.M. Zhang, K.W. Xu, Generalized stacking fault energy in FCC metals with MEAM, Appl. Surf. Sci. 254(5) (2007) 1489-1492.

[15]X. Z. Wu, R. Wang, S. F. Wang, Generalized-stacking-fault energy and surface properties for HCP metals: A first-principles study, Appl. Surf. Sci. 256(11) (2010) 3409-3412.

[16] X. Z. Wu , R. Wang, S. F. Wang, Ab initio calculations of generalized-stacking-fault energy surfaces and surface energies for FCC metals, Appl. Surf. Sci. 256(21) (2010) 6345-6349.

[17] S. F. Wang, H. L. Zhang, X. Z. Wu and R. P. Liu, J. Phys. Condens. Matter 22 055801(2010).

[18] P. E. Blöchl. Projector augmented-wave method, Phys. Rev. B. 50 (1994) 17953.

[19] G. Kresse, D. Joubert. From ultrasoft pseudopotentials to the projector augmented-wave method Phys. Rev. B. 59 (1999) 1758.

[20]G. Kresse, J. Hafner, Ab initio molecular dynamics for open-shell transition metals, Phys. Rev. B. 48 (1993) 13115.

[21]G. Kresse, J. Furthmller, Efficiency of ab-initio total energy calculations for metals and semiconductors using a plane-wave basis set, Comput. Mater. Sci. 6 (1996) 15-50.

[22] G. Kresse, J. Furthmller, Efficient iterative schemes for ab initio total-energy calculations using a plane-wave basis set, Phys. Rev. B. 54 (1996) 11169.

[23] S. B. Zhang and M. L. Cohen. High-pressure phases of III-V zinc-blende semiconductors, Phys. Rev. B. 35 (1987) 7604.

[24]R. W. G. Wyckoff, Crystal Structures (Interscience, New York), 1 (1963).

[25] R. M. Martin, Elastic Properties of ZnS Structure Semiconductors, Phys. Rev. B. 1 (1970) 4005.

[26] R. I. Cottam, G. A. Saunders. The elastic constants of GaAs from 2 K to 320 K, J. Phys. C. 6(15) (1973) 2105-2118.

[27] J. P. Hirth, J. Lothe, Theory of Dislocations 2nd ed (New York: Wiley) (1982), 373.

[28] J. W. Christian, V. Vitek. Dislocations and stacking faults, Rep. Prog. Phys. 33(1) (1970) 307-412. 